\title{
THIS IS A TEST
}

CATHERINE LU

BAM! Youth Slam

\section{ABSTRACT}

\section{KEYWORDS}

education, spoken

This spoken word poem is targeted towards anyone affected by the systematic education that continues all throughout our lives. It brings the truth out, of how this is all a cycle of testers word and testing.

\section{$\underline{\text { AUDIO LIN }}$}

This is a test.

T R A N S C R I P T

Room 42, high noon, you will be tested on how well you perform on tests because you were taught how to go to school where you learned how to learn for tests testing tests in school schooling how to school teaching how to be taught for tests testing tests in school schooling school teaching how to be taught.

So test me.

Test my patience.

Test my temper.

My limits, held together by

Dermatologist-tested duct tape, chef-tasted rubber, toast my tyrant on repeat for quality demonstration, warranty recommendation, I am a tested product with a clear background check. I am a tested product with great credit history.

"Pick a number, queue in. Patient

$$
\text { [2-4-6-F] }
$$

how much

[pain]

do you feel on a scale of 1 to $10 ? "$

\section{Can I respond with a haiku?}

Emotional pain

constricts its barbed wire a-

round long long healed scars.

No? Abstract answers not allowed!? On a scale of 1-10, rate my existence.

On a scale of 1-10, with one decimal point, rate. . . your mom.

On a scale of 1-10, with one decimal point. . . get it right, judges! Drum roll, please! 
Score. .. the meaning of life. And score the expression on your face right now.

Measuring cup our capability of spiritual tolerance. Does empathy stay buoyant at 38 Celsius? On a scale of $1-10$, rate

my essence

of existence that is

poetry.

Self learning algorithms can't get it right. I'm only human, neither can I

even-

the test makers can't score perfect.

When the tested graduate to testers, they do what they've been taught.

We are as flawed as the system that made us that made the system that made us that made systematically standardized testing, systematically sorting, boxing a hundred billion neurons for witness testimony. . .

so test me.

I am a licensed product with tested references.

I am tested goods with a PhD in acronyms.

I am a qualified human resource, as in Human resource.

My mouth is certified, sealed, and stamped shut.

I finally got a test to see if I know when all the testing stops and I am finally ready to use those results without studying for another - that was a test.

A test?

This is.

A test.

\section{AUTHOR COMMENTARY}

In the Ontario education system, I have been standardized tested since Grade 3's EQAO (Education Quality and Accountability Office, an independent, government-funded agency that does large-scale tests to measure learning) test. And the paragraphs in our report cards mean less than the first four letters of the alphabet.

I am currently in my last year of high school, and the education system has my creative personality boxed to its limits. English class is my worst subject, but Writer's Craft (thanks to a more flexible teacher) is my best.

The different creative learning styles of students, when transferred to exams and unit tests, become a numerical average 
given to universities. No admissions officer will look twice at a student below the passing grade, even if only one course is dragging them down. The experience of being a number has in part compelled me to write this poem in protest. At poetry slams,

The experiences of being a number has in part compelled me to write this poem in protest.

competitors are graded only by a score on a scale of one to ten with one decimal point, and on some occasions the judging is biased too. But points are not the point, poetry is the point of spoken word slams.

Moving on, I want to thank and give credit to Patrick de Belen, an artist educator and my spoken word mentor. I think he shares a point of view with me on the inflexibility of being a number in the largest school board in Canada, as I have heard many poems he has written about school, learning, and children. Teaching with art as a medium has turned the lives of students around, especially in 'Poetry Saved Our Lives', a library-run program where we first met.

Find him on many social media platforms, like YouTube and his website here: http://www.patrickdebelen.com/ 